\title{
On the Extent of the Existence of Broad Line Region in Seyfert Galaxies
}

\author{
Oyor David Ifeanyichukwu ${ }^{1, *}$, Ogwo Jemima Ngozi ${ }^{2}$ \\ ${ }^{1}$ Department of Science Laboratory Technology, Akanu Ibiam Federal Polytechnic, Afikpo, Nigeria \\ ${ }^{2}$ Department of Physics, Abia State University, Uturu, Nigeria
}

\section{Email address:}

oyordavid $@$ yahoo.com (O. D. Ifeanyichukwu), jimogwo@yahoo.com (O. J. Ngozi)

${ }^{*}$ Corresponding author

\section{To cite this article:}

Oyor David Ifeanyichukwu, Ogwo Jemima Ngozi. On the Extent of the Existence of Broad Line Region in Seyfert Galaxies. American Journal of Astronomy and Astrophysics. Vol. 7, No. 2, 2019, pp. 33-38. doi: 10.11648/j.ajaa.20190702.12

Received: July 29, 2019; Accepted: September 21, 2019; Published: October 9, 2019

\begin{abstract}
We present statistical results from a very large sample of Seyfert galaxies (217,272 galaxies) obtained from SDSS DR10 (Sloan Digital Sky Survey Data Release 10). From their observed parameters which includes; flux of hydrogen alpha $(\mathrm{H} \alpha)$, luminosity distance and velocity dispersion, we computed other relevant parameters such as; bolometric luminosity, black hole mass and mass accretion rate, which enabled us classify these Seyfert galaxies into Seyfert 1s (188,486 galaxies) and Seyfert 2s (28,786 galaxies). Analyses on these computed parameters revealed that Seyfert 2 galaxies were less luminous, more massive and accrete less matter than the Seyfert 1 galaxies. Further analysis on Seyfert 2s based on their mass accretion rate led to their classification into hidden broad line region (HBLR) Seyfert 2s (12,988 galaxies) and non-hidden broad line region (non-HBLR) Seyfert 2s (15,798 galaxies) which shows that, the HBLR S2s accrete more matter than the non-HBLR S2s. All the results obtained suggest that the bolometric luminosity alone is not sufficient in determining the extent of the existence of the BLR in Seyfert 2 galaxies rather the mass accretion rate should be taking into consideration in determining the appearance and disappearance of the BLR.
\end{abstract}

Keywords: Galaxies, Seyfert, Accretion Rate, Bolometric Luminosity and Broad Line Region

\section{Introduction}

Seyfert galaxies are a subclass of active galactic nuclei (AGN) and are categorized as low luminosity, radio-quiet AGN, hosted in spiral or lenticular galaxies [1]. They have an absolute B-band luminosity of $M_{B}>-21.51+5 \log h_{0}$ [2], with $h_{0}$ denoting the Hubble constant in units of $100 \mathrm{~km}$ $\mathrm{s}^{-1} \mathrm{Mpc}^{-1}$. Seyfert galaxies are one of the largest groups of active galaxies, along with quasars. They have similar nucleus like quasars (very luminous, distant and bright sources of electromagnetic radiation) with very high surface brightness whose spectra reveal strong, high-ionization emission lines [3], but unlike quasars, their host galaxies are clearly detectable $[4,5]$.

They have super massive black hole (SMBH) at their centers which are surrounded by accretion discs of in-falling materials. The accretion discs are believed to be the source of the observed ultraviolet (UV) radiation which provides the best diagnostic for the composition of the surrounding material [6]. It is the active galactic nuclei in Seyfert galaxies that produce the strong radiation which excites gas around the central regions, giving rise to the observed emission lines [7]. The emission lines seen on the spectrum of Seyfert galaxies comes either from the surface of the accretion disc itself, or from clouds of gas illuminated by the central engine in an ionization cone. The exact geometry of the emitting region is difficult to determine due to poor resolution of the galactic center. However, each part of the accretion disc has a different velocity relative to our line of sight, and the faster the gas is rotating around the black hole, the broader the emission line will be [8].

In gathering the spectrophotometric data for the Seyfert galaxies, it became obvious that not all spectra from Seyfert galaxies are identical. Seyfert galaxies were therefore classified according to the characteristics of their emission spectra as; Seyfert 1 galaxies (S1s) showing both broad and 
narrow emission lines and Seyfert 2 galaxies (S2s) showing only narrow emission lines $[8,9]$. The detection of broad emission lines in the spectropolarimetric observations of few Seyfert 2 galaxies laid the foundation for the Seyfert unification scheme [10]. The unification scheme postulates that the same engine is at work in all kinds of Seyfert galaxies [9], that S1s and S2s constitute the same parent population, and their difference in appearance is exclusively because of orientation effects and anisotropic obscuration by optically thick material, possibly distributed in a toroidal geometry around the AGN. In Seyfert 2s, the dusty torus intercepts the observer's line-of-sight and blocks the direct view of the broad line region and accreting black hole. While, in S1s, the observer's line-of-sight is away from the obscuring torus, and thus, broad line region and accreting black hole are directly visible [10-12].

\section{Method}

The data used in this paper were obtained from SDSS DR10 (Sloan Digital Sky Survey Data Release 10), the paper for it is Ahn et al., submitted to APJS and posted at arXiv: 1307.7735 [13]. The data consist of 217,272 Seyfert galaxies, with their observed parameters which includes; flux of hydrogen alpha $(\mathrm{H} \alpha)$, velocity dispersion and luminosity distance. Based on the flux of hydrogen alpha $(\mathrm{H} \alpha)$, the bolometric luminosity $\left(\mathrm{L}_{\mathrm{bol}}\right)$ was calculated and further, the mass accretion rate $(\dot{m})$ was deduced. The black hole mass was calculated from their velocity dispersion using the $\mathrm{M}-$ sigma relationship. Based on their bolometric luminosities; $\mathrm{L}_{\text {bol }}>\mathrm{L}_{\text {bol(threshold) }}=$ Seyfert $1 \mathrm{~s}$, while $\mathrm{L}_{\text {bol }} \leq \mathrm{L}_{\text {bol(threshold) }}=$ Seyfert 2s. The Seyfert 2 galaxies were further classified into hidden broad line region (HBLR) and non-hidden broad line region (non-HBLR) Seyfert 2 galaxies based on their accretion rate $(\dot{\mathrm{m}}) ; \dot{\mathrm{m}}>\dot{\mathrm{m}}_{\min }=$ HBLR, while $\dot{\mathrm{m}} \leq \dot{\mathrm{m}}_{\min }=$ nonHBLR.

\section{Analysis and Results}

We estimated the luminosity of the narrow $\mathrm{H} \alpha$ line from the flux of hydrogen alpha and its luminosity distance $\left(D_{L}\right)$ using the empirical relation:

$$
\mathrm{F}=\mathrm{L} / 4 \pi \mathrm{D}_{\mathrm{L}}^{2}
$$

Using the $M-\operatorname{sigma}(M-\sigma)$ relation, the mass of the supermassive black hole for the Seyfert galaxies was estimated,

$$
\mathrm{M}_{\mathrm{BH}}=10^{8} \mathrm{M}_{\odot} \times 3.1\left(\sigma / 200 \mathrm{kms}^{-1}\right)^{4}
$$

where; $\mathrm{M}_{\mathrm{BH}}$ is the black hole mass, $\mathrm{M}_{\odot}$ is the solar mass and $\sigma$ is the velocity dispersion. The log distributions of black hole mass for the Seyfert galaxies are shown in figures $1-3$. Black hole mass distributions for all the Seyfert galaxies show a mean $\log$ of $8.486\left(\mathrm{M}_{\mathrm{BH}}=3.062 \times 10^{8} \mathrm{M}_{\odot}\right)$ with $\log$ of standard deviation 0.6022 as shown in figure 1 . The Seyfert $1 \mathrm{~s}$ and 2 s (figures 2 and 3 ) have mean of their $\log$ of black hole mass as $8.395\left(\mathrm{M}_{\mathrm{BH}}=2.483 \times 10^{8} \mathrm{M}_{\odot}\right)$ and 9.078 $\left(\mathrm{M}_{\mathrm{BH}}=1.197 \times 10^{9} \mathrm{M}_{\odot}\right)$ with $\log$ of standard deviations 0.5606 and 0.5230 respectively. A review of the $\log$ distributions of black hole mass for Seyfert 1s and 2s shows that Seyfert $2 \mathrm{~s}$ are more massive than the Seyfert 1s.

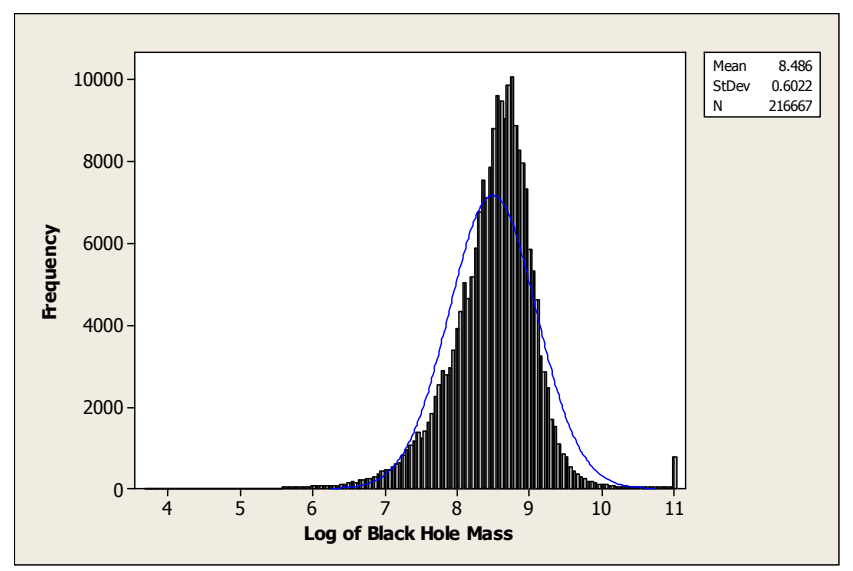

Figure 1. Distribution of logarithm of Black Hole Mass for both Seyfert 1 and Seyfert 2 galaxies.

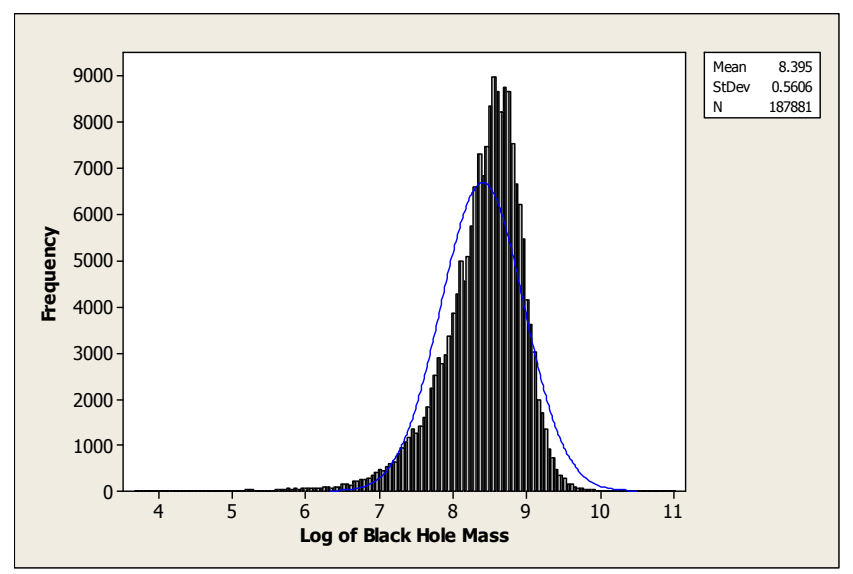

Figure 2. Distribution of logarithm of Black Hole Mass for Seyfert 1 galaxies.

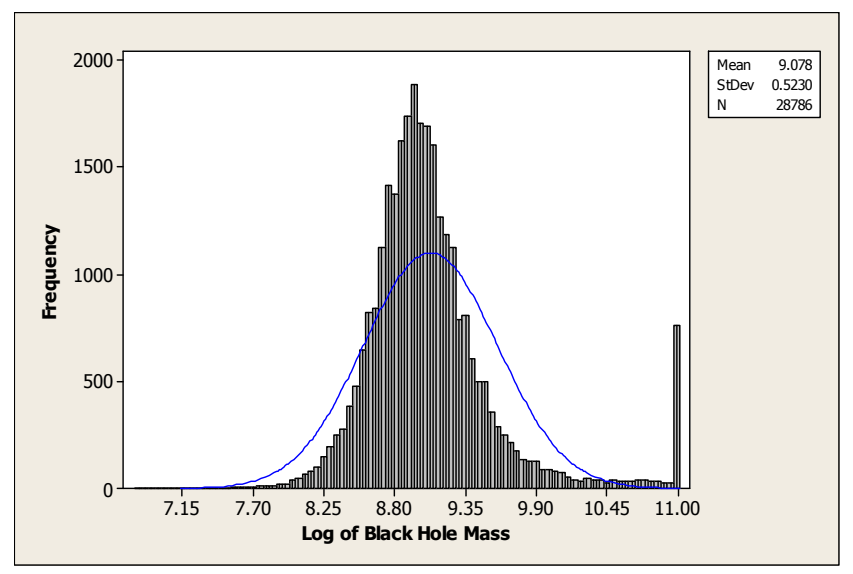

Figure 3. Distribution of logarithm of Black Hole Mass for Seyfert 2 galaxies.

The bolometric luminosity $\left(\mathrm{L}_{\mathrm{bol}}\right)$ which serves as an index in the classification of the galaxies into Seyfert 1 and Seyfert 
2 galaxies was calculated for the sources from the luminosity of the narrow $\mathrm{H} \alpha$ line;

$$
\log \mathrm{L}_{\mathrm{bol}}=1.176 \log \mathrm{L}(\mathrm{H} \alpha)-4.91
$$

According to Elitzur et al., there exist a threshold bolometric luminosity which determines the appearance and disappearance of the broad line region (BLR). This is given by, $\mathrm{L}_{\text {bol(threshold) }}=5$ $\mathrm{x} 10^{39} \mathrm{~m}^{2 / 3} \mathrm{ergs}^{-1}$, where $\mathrm{m}=\mathrm{M}_{\mathrm{BH}} / 10^{7} \mathrm{M}_{\odot}$, and $\mathrm{M}_{\mathrm{BH}}$ is the black hole mass of the object and $\mathrm{M}_{\odot}$ is the mass of the Sun [14]. Seyfert galaxies with bolometric luminosities greater than the threshold bolometric luminosity were classified as Seyfert 1 galaxies (S1s) (188,486 galaxies) while those less than or equal the threshold bolometric luminosity were classified as the Seyfert 2 galaxies (S2s) (28,786 galaxies).

The distribution of logarithm of the bolometric luminosity of the Seyfert galaxies are given in figures $4-6$. There is a noticeable difference between the mean of log of bolometric luminosities of the Seyfert galaxies. The distribution of the whole sample is shown in figure 4 , with mean log of bolometric luminosity 44.12 and standard deviation of 0.9830 . The Seyfert 1s and $2 \mathrm{~s}$ (figures 5 and 6 ) have the mean $\log$ of their bolometric luminosity as 44.25 and 43.31 with standard deviations 0.8579 and 1.305 respectively. These results indicate that the Seyfert $1 \mathrm{~s}$ are more luminous than the Seyfert $2 \mathrm{~s}$.

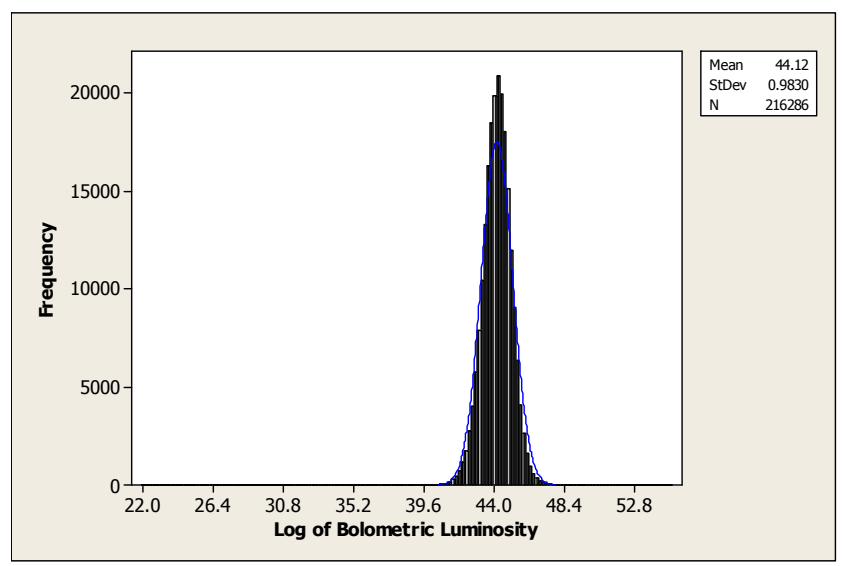

Figure 4. Distribution of logarithm of Bolometric Luminosity for both Seyfert 1 and Seyfert 2 galaxies.

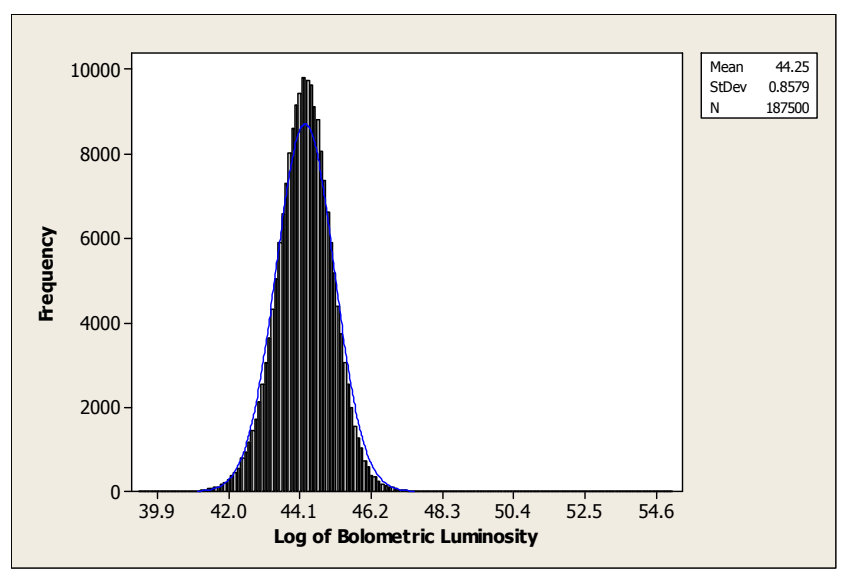

Figure 5. Distribution of logarithm of Bolometric Luminosity for Seyfert 1 galaxies.

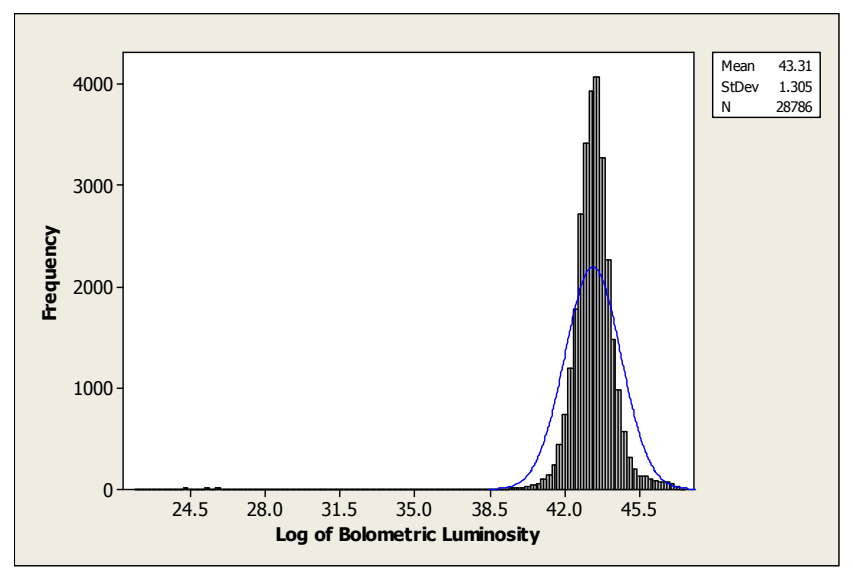

Figure 6. Distribution of logarithm of Bolometric Luminosity for Seyfert 2 galaxies.

After the classification, we calculated the mass accretion rate $(\dot{m})$ for the source and equally for each of the class of the Seyfert galaxies using the relation between mass accretion rate, bolometric luminosity $\left(\mathrm{L}_{\mathrm{bol}}\right)$ and velocity dispersion $(\sigma)$ as postulated by Laor [15]:

$$
\log \dot{\mathrm{m}}=-36.977+\log \mathrm{L}_{\mathrm{bol}}-4.02 \log \sigma
$$

The $\log$ distributions of accretion rates for the Seyfert galaxies are shown in figures $7-9$. Accretion rate distributions for all the Seyfert galaxies show a mean log of 2.101 (accretion rate, $\dot{\mathrm{m}}=0.0079$ ) with standard deviation 1.089 as shown in figure 7. The Seyfert 1s and 2s (figures 8 and 9) have the mean $\log$ of their accretion rate as -1.884 (m $=0.0131)$ and $-3.508(\dot{\mathrm{m}}=0.0003146)$ with standard deviations 0.9127 and 1.092 respectively. An assessment of the $\log$ distributions of accretion rates for Seyfert 1s and $2 \mathrm{~s}$ shows that Seyfert 1s have higher accretion rate than those of Seyfert 2s. This is in agreement with the postulate of Elitzur et al. that, the decrease in accretion rate is as a result of the increment on the black hole mass and the prediction of the presence of the obscuring torus and BLR disappearance at a low accretion rate [14].

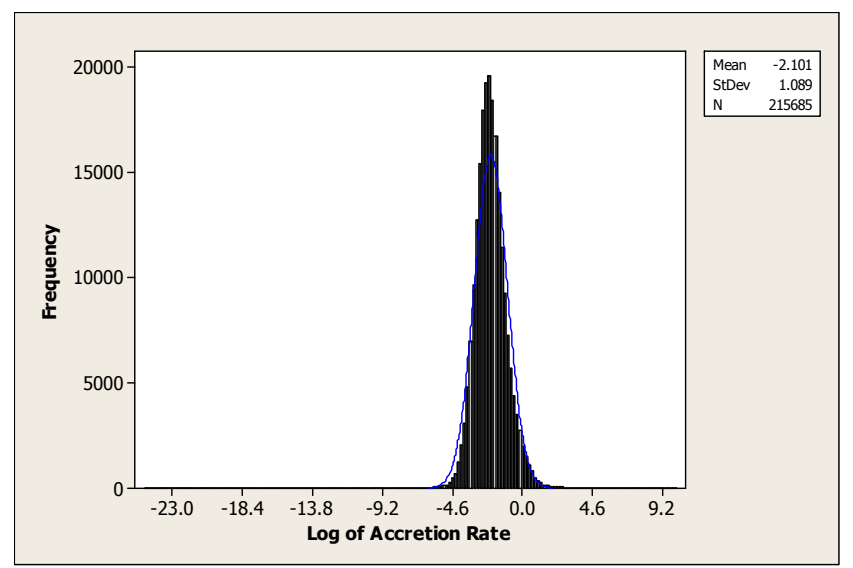

Figure 7. Distribution of logarithm of Accretion Rate for both Seyfert 1 and Seyfert 2 galaxies. 


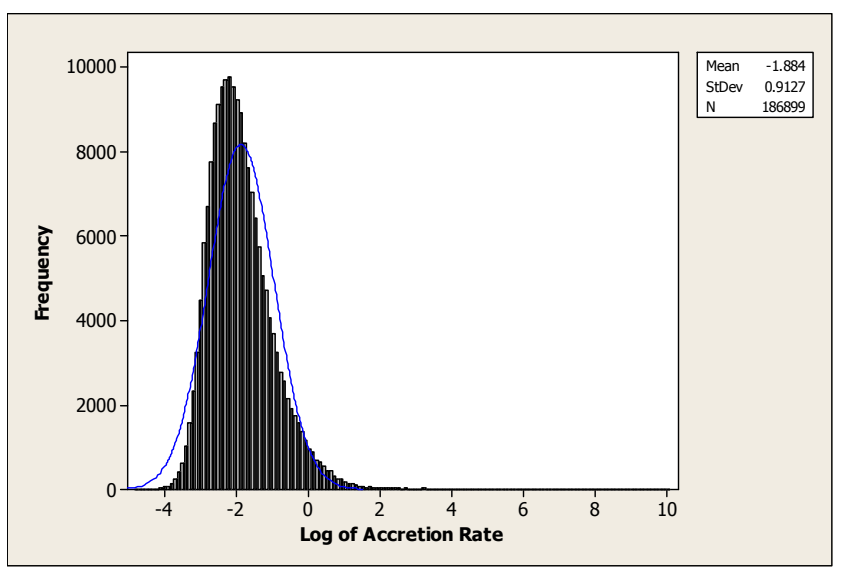

Figure 8. Distribution of logarithm of Accretion Rate for Seyfert 1 galaxies.

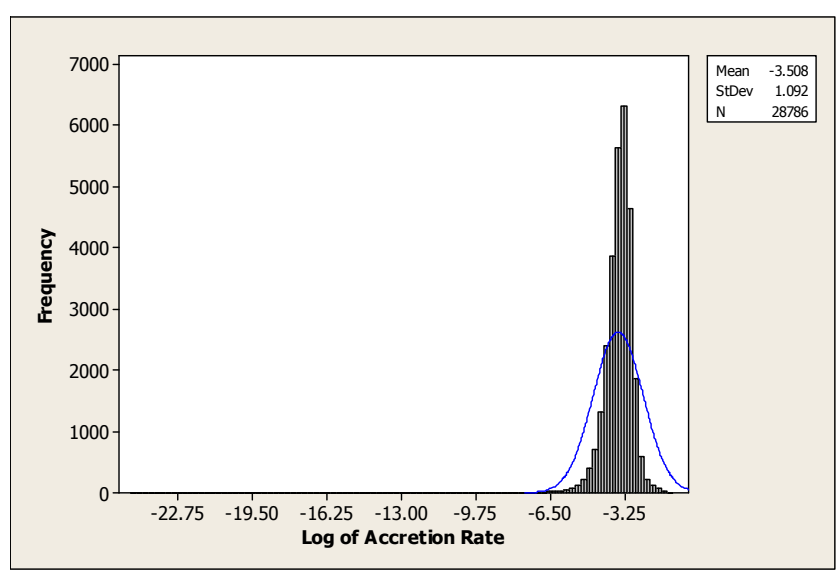

Figure 9. Distribution of logarithm of Accretion Rate for Seyfert 2 galaxies.

To further probe the existence of the BLR, we analyzed the Seyfert $2 \mathrm{~s}$ according to Laor, who indicated that the absence of a BLR in some objects cannot be just due to very low values of the bolometric luminosity $\left(\mathrm{L}_{\mathrm{bol}}\right)$, for example, NGC 4395 is a type $1 \mathrm{AGN}$ with $\mathrm{L}_{\text {bol }}$ as low as $\sim 1.9 \times 10^{40}$ $\operatorname{ergs}^{-1}$ [15]. According to him, there is a minimum accretion rate $\left(\dot{\mathrm{m}}_{\min }\right)$ below which the BLR will not survive and this minimum accretion rate is related to the black hole mass $\left(\mathrm{M}_{\mathrm{BH}}\right)$ by this equation;

$$
\dot{\mathrm{m}}_{\min }=10^{-12.3} \mathrm{M}_{\mathrm{BH}}
$$

We observed that there exist a BLR in some S2s, those with $\dot{\mathrm{m}}>\dot{\mathrm{m}}_{\min }$ were classified as the hidden broad line region (HBLR) S2s (12,988 galaxies), while those with $\dot{\mathrm{m}} \leq \dot{\mathrm{m}}_{\min }$ are the non-hidden broad line region (non-HBLR) S2s $(15,798$ galaxies).

The $\log$ distributions of accretion rates for the Seyfert 2 galaxies are shown in figures $10-12$. Accretion rate distributions for all the Seyfert 2 galaxies show a mean $\log$ of $-3.508(\dot{\mathrm{m}}=0.0003146)$ with standard deviation 1.092 as shown in figure 10. The HBLR and non-HBLR Seyfert $2 \mathrm{~s}$ (figures 11 and 12) have the mean $\log$ of their accretion rate as $-3.187(\dot{\mathrm{m}}=0.0006501)$ and $-3.773(\dot{\mathrm{m}}=0.0001687)$ with standard deviations 0.3358 and 1.388 respectively. An assessment of the $\log$ distribution of accretion rates for HBLR and non-HBLR Seyfert 2s shows that HBLR Seyfert
$2 \mathrm{~s}$ have higher accretion rate than the non-HBLR Seyfert $2 \mathrm{~s}$.

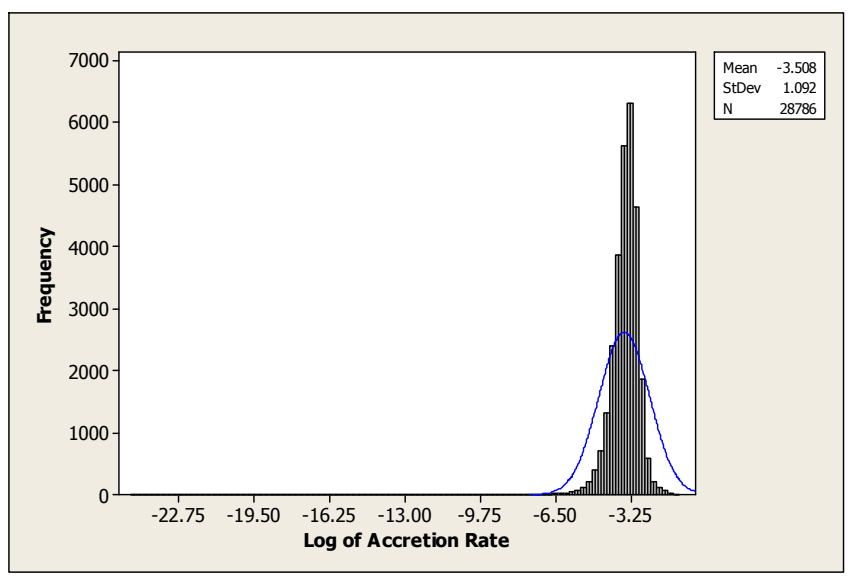

Figure 10. Distribution of logarithm of Accretion Rate for Seyfert 2 galaxies.

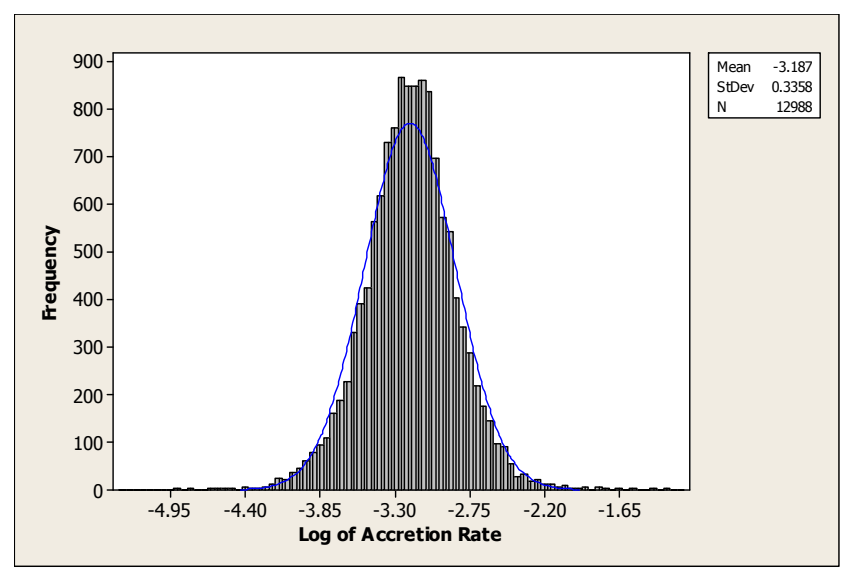

Figure 11. Distribution of logarithm of Accretion Rate for HBLR Seyfert 2 galaxies.

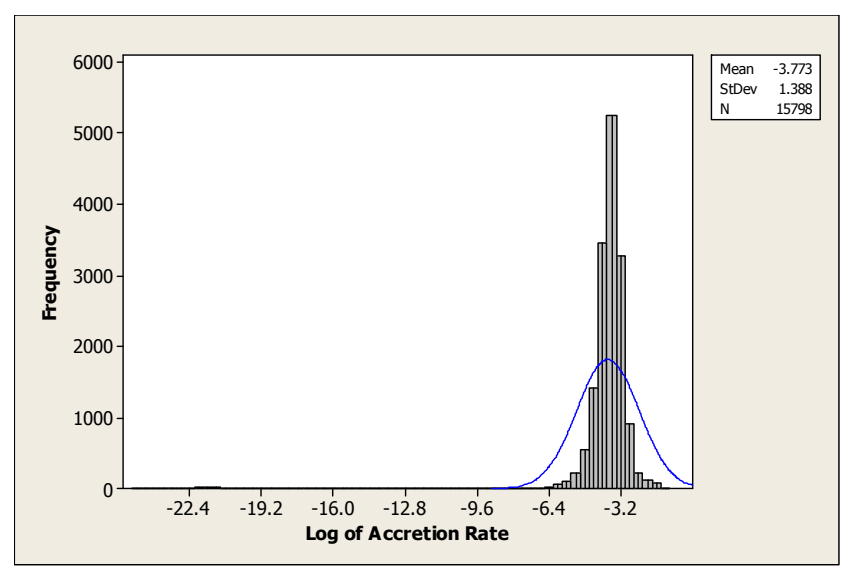

Figure 12. Distribution of logarithm of Accretion Rate for non-HBLR Seyfert 2 galaxies.

\section{Discussion and Conclusion}

Table 1 shows distribution statistics for the parameters and Table 2 is the correlation values for all the Seyfert galaxies ( $\left.\mathrm{S}_{\mathrm{ALL}}\right)(217,272$ galaxies), the Seyfert 1 galaxies (S1s) $(188,486$ galaxies) and the Seyfert 2 galaxies (S2s) (28,786 galaxies). 
Table 1. Distributions of Parameters for the Seyfert Galaxies.

\begin{tabular}{lllllll}
\hline \multirow{2}{*}{$\begin{array}{l}\text { Paramete } \\
\text { rs }\end{array}$} & Mean & & \multicolumn{4}{c}{ Standard Deviation } \\
\cline { 2 - 7 } & $\mathbf{S}_{\text {ALL }}$ & S1s & S2s & $\mathbf{S}_{\text {ALL }}$ & S1s & S2s \\
\hline $\mathrm{L}$ & 44.121 & 44.246 & 43.309 & 0.983 & 0.858 & 1.305 \\
$\dot{\mathrm{m}}$ & 0.0079 & 0.0131 & 0.000315 & 1.089 & 0.9127 & 1.092 \\
$\mathrm{Log} \mathrm{M}$ & 8.486 & 8.395 & 9.078 & 0.6022 & 0.5606 & 0.5230 \\
\hline
\end{tabular}

Table 2. Correlations of Parameters for the Seyfert Galaxies.

\begin{tabular}{llll}
\hline Variables & $\mathbf{S}_{\mathrm{ALL}}$ & S1s & S2s \\
\hline$\dot{\mathrm{m}}$ and $\mathrm{L}_{\mathrm{bol}}$ & 0.042 & 0.051 & 0.383 \\
$\mathrm{M}_{\mathrm{BH}}$ and $\dot{\mathrm{m}}$ & -0.008 & -0.008 & 0.394 \\
$\mathrm{M}_{\mathrm{BH}}$ and $\mathrm{L}_{\text {bol }}$ & 0.122 & 0.226 & 0.573 \\
\hline
\end{tabular}

From the distributions, we noticed a distinct difference in the mean values of the bolometric luminosity, the black hole mass, and the accretion rate between Seyfert $1 \mathrm{~s}$ and $2 \mathrm{~s}$. This reveals the nature of Seyfert $2 \mathrm{~s}$ as being more massive with less accretion rate than the Seyfert 1s. Our results show that, as the black hole mass of an AGN (Seyfert galaxy) increases, there is a decrease in its bolometric luminosity and accretion rate and the region of broad line emission progressively declines from its high intensity in Seyfert 1s to the point of disappearance at sufficiently low bolometric luminosity (Seyfert 2s). Contrary to the standard unification scheme which posits that, the torus obscuration and the observer location are the only factors in determining the spectral class of any given AGN; it is possible that intrinsic broad line emission from Seyfert galaxies may follow an evolutionary trend from Seyfert 1 to Seyfert 2 as accretion unto the supermassive black hole is decreasing.

Table 3. Distributions of Parameters for the Seyfert 2 Galaxies.

\begin{tabular}{llllll}
\hline \multirow{2}{*}{ Parameters } & Mean & & \multicolumn{2}{c}{ Standard Deviation } \\
\cline { 2 - 6 } & S2s & HBLR S2s & Non-HBLR S2s & S2s & HBLR S2s \\
\hline $\mathrm{L}_{\text {bol }}$ & 43.31 & 43.47 & 43.18 & 1.305 & 0.6596 \\
$\dot{\mathrm{m}}$ & $3.15 \mathrm{E}-4$ & $6.501 \mathrm{E}-4$ & $1.687 \mathrm{E}-4$ & 1.092 & 0.3358 \\
$\log \mathrm{M}_{\mathrm{BH}}$ & 9.078 & 8.920 & 9.208 & 0.6022 & 0.3303 \\
\hline
\end{tabular}

Table 4. Correlations of Parameters for the Seyfert 2 Galaxies.

\begin{tabular}{llll}
\hline Variables & S2s & HBLR S2s & Non-HBLR S2s \\
\hline m and $\mathrm{L}_{\text {bol }}$ & 0.383 & 0.600 & 0.337 \\
$\mathrm{M}_{\mathrm{BH}}$ and $\dot{\mathrm{m}}$ & 0.394 & 0.586 & 0.395 \\
$\mathrm{M}_{\mathrm{BH}}$ and $\mathrm{L}_{\text {bol }}$ & 0.573 & 0.988 & 0.573 \\
\hline
\end{tabular}

A cursory look at Table 3 shows that the bolometric luminosity and accretion rate of HBLR S2s are higher than those of non-HBLR S2s, while the black hole mass are higher in non-HBLR S2s than in HBLR S2s. The similarity in the correlation results of the Seyfert 2 galaxies and the non-HBLR Seyfert 2 galaxies in Table 4, suggests that the non-HBLR Seyfert 2 galaxies are the 'true' Seyfert 2 galaxies, and this agrees with the postulate of Pappa et al., in the existence of the true Seyfert 2 galaxies [16].

\section{Conclusion}

From our findings, the bolometric luminosities of Seyfert 2 s fall below the minimum bolometric luminosity in which the BLR ought not to exist. But further analysis using the accretion rate revealed that, there are some of the Seyfert 2 galaxies that show evidence of the existence of the BLR. That is, having accretion rate greater than the minimum accretion rate even at low bolometric luminosity. In line with this development, we conclude that the bolometric luminosity alone is not sufficient in determining the extent of the existence of the BLR in Seyfert 2 galaxies rather the accretion rate should be taking into consideration in determining the appearance and disappearance of the BLR.

\section{References}

[1] Singh, V., Shastri, P., Risaliti G. (2011). Measuring the Level of Nuclear Activity in Seyfert Galaxies and the Unification Scheme. A \& A, 532, A84.

[2] Schmidt, M. and Green, R. F. (1983). Quasar Evolution derived from the Palomar Bright Quasar Survey and other Complete Quasar Surveys. ApJ, 269, 352.

[3] Peterson, B. M. (1997). An Introduction to Active Galactic Nuclei. Cambridge University Press.

[4] Maiolino, R. and Rieke, G. H. (1995). Low-Luminosity and Obscured Seyfert Nuclei in Nearby Galaxies. ApJ, 454; 95-105.

[5] Petrov, G. T. (2004) (ed). Active Galaxy Nuclei. Bulgarian Academy of Science/Institute of Astronomy.

[6] Davidsen, A. F. (1993). Far-ultraviolet Astronomy on the Astro-1 Space Shuttle Mission. Science, 259 (5093), 327-334.

[7] Ridpath, I. (1997). Dictionary of Astronomy. Oxford University Press. United Kingdom.

[8] Goad, M. R., Korista, K. T., and Ruff, A. J. (2012). The Broad Emission Line Region: The Confluence of the Outer Accretion Disc with the Inner Edge of the Dusty Torus. MNRAS, 426 (4), 3086-3111.

[9] Ricci, C., Walter, R., Courvoisier, T. J.-L., and Paltani, S. (2011). Reflection in Seyfert Galaxies and the Unified Model of AGN. A \& A, 532, A102.

[10] Antonucci, R. R. J. and Miller, J. S. (1985). Spectropolarimetry and the Nature of NGC 1068. ApJ, 297, 621-632.

[11] Antonucci, R. R. J. (1993). Unified Models for Active Galactic Nuclei and Quasars. ARA \& A, 31, 473. 
[12] Singh, V., Shastri, P., Ishwara-Chandra, C. H., and Athreya, R. (2013). Low Frequency Radio Observations of Seyfert Galaxies: A Test of the Unification Scheme. A \& A, 554, A85.

[13] Ahn, C. P., Alexandroff, R., Allende, Prieto C., et. al., (2013). The Tenth Data Release of the Sloan Digital Sky Survey: First Spectroscopic Data from the SDSS-III Apache Point Observatory Galactic Evolution Experiment. ApJ, arXiv: 1307.7735

[14] Elitzur, M., Ho, L. C., and Trump, J. R. (2014). Evolution of Broad-Line Emission from Active Galactic Nuclei. MNRAS, 438, 3340-3351.
[15] Laor, A. (2003). On the Nature of Low Luminosity Narrow Line AGN. ApJ, 590, 86.

[16] Pappa, A., Georgantopoulos, I., Stewart G. C., and Zezas, A. L. (2001). The X-ray Spectra of Optically Selected Seyfert 2 galaxies: Are there any Sy2 Galaxies with no Absorption? MNRAS, arXiv: astro-ph/0104061. 